\title{
Technology of UWB Communication System Synchronization Capture
}

\author{
ZHOU Yangyang ${ }^{1, a}$, XIE Xusheng ${ }^{1, b}$ \\ ${ }^{1}$ College of Computer Information Engineering, Jiangxi Normal \\ University, Nanchang, China \\ anc0801@163.com, bushengxie@163.com
}

\begin{abstract}
UWB is a high data transfer rate, high-performance, low-cost, low-power short-range wireless communications technology data transmission, but it is narrow in the use of non-sinusoidal pulse high-speed transmission of data , but also faced with many problems. Based on the characteristics of UWB communications, we propose an adaptive synchronization acquisition algorithms, fully overcome the disadvantages of multi- path channel , to quickly capture the sync signal, and access to relevant synchronization information, reducing the transmission bit rate, at an average acquisition time and channel estimation on the more obvious advantages.
\end{abstract}

Key words: UWB; Adaptive; Synchronous capture

\section{Introduction}

UWB (Ultra Wide Band) is a non- carrier communication technology, using ns to us -grade non-sinusoidal narrow pulses to transmit data ${ }^{[1][2][3]}$. UWB communications as with the conventional digital radio communication must meet the requirements of digital communication , that is, to ensure consistency in the receiving end of the signal frequency and phase. According to the characteristics of UWB communications technology , non- sinusoidal pulse transmission narrow its use of data , and because narrower pulse signal as an information carrier ( no carrier directly send information), even if there is a small synchronization error will also result in the reception produce more significant errors in the signal, so that the performance of the receiver is greatly reduced. Conventional narrowband communication technology is commonly used ideal local carrier signal of the received synchronization sliding correlation , it is apparent that this method has a pulse width limit for UWB communications , it will cause great difficulty. Conventional synchronization algorithm is based on the correlation detection on the matched filtering and adjusting the receiver transmitting side transmits a known signal waveform , generating a noise-free reference signal. When not agreed in advance, the receiver receives the signal transmitted by the transmitter , the received signal and the signal related to the standard template , after adjusting the relevant parameters related to the output 
delay time when the correlation value reaches a threshold value is obtained by the synchronization signal acquisition time . UWB communication cannot adapt to the conventional acquisition algorithm , the reason that the signal transmitted UWB communication nanosecond narrow pulse, so that the receiver will continue to search for matching a long -consuming valuable time ; Secondly, because of the narrow pulse signal source UWB taken under conditions of multipath channel , the receiver cannot determine the shortest path to reach the signal is strongest, so there may be more than meets the synchronization signal arrives access conditions, resulting in the receiver cannot determine the correct signal or reduce the signal receiver detects the reception rate and sensitivity ${ }^{[4][5]}$. In order to solve the above problems , this paper proposes a novel algorithm for adaptive synchronization capture and simulation to be realized.

\section{Technology of Communication System Synchronization Capture}

For the study of communication and information currently synchronizing access to technology are many. There are existing search algorithms typically four kinds : linear search method ${ }^{[6]}$, M unit hopping search method , bit flipping binary search algorithm and Binary search method. These search methods can reduce the average acquisition time and increase the probability of capturing a certain extent, but to a greater need to study improvements to enhance other methods , some researchers have conducted a number of columns based on artificial neural networks Related research, but its own characteristics make little progress

Chip synchronization with the PN code synchronization capture technology , refers to a signal waveform correlation function within the local sample using cycle -related features will receive the chip period, and then use the sample function recursive method amended several times, is also aimed at to reduce the effects of noise , such as the local signal can be associated with the received signal to match , the reception signal to reduce the energy loss , when it reaches a relatively accurate synchronization chip timing, based on the known chip synchronization signal using the $\mathrm{PN}$ code is a sliding correlation rapid completion of PN code acquisition. Disadvantages associated with the PN chip synchronization code synchronization acquisition technique is also evident that to get the start time of the received signal in the case of multipath and multiple access interference, and various noise, to be able to reduce the bit error rate , which is a hardware circuit design requires relatively high. Acquire synchronization using correlation detection , the receiver and the transmitter if the agreement is good signal waveform, and generates a local signal of the standard templates, we received signal with a local template correlation signal and adjust the delay time, when the correlation value reaches the output maximum or exceeds the threshold, it is related to the delay time signal synchronization acquisition time. However, if used for UWB, the algorithm , there are two serious problems will not be applied to UWB communication, namely: (1)The duration of the pulse UWB signal sources using only a few ns, 
the receiver searches will be very frequent, time will consume a lot of resources; (2)UWB single pulse signal in a multipath channel, through reflection, scattering, to reach the receiver has become a multi-pulse signals of unknown composition , and the energy will be dispersed in the diameter, but the diameter of the first arrival of energy is not necessarily to carry the largest component , then there will be more of the same signal multipath component that we have satisfied the conditions of synchronization acquisition will greatly reduce the probability of detection of the signal receiver. To this end , this paper presents an adaptive synchronization acquisition under multipath channel based algorithm , the first use multipath environment characteristics to construct a local standard template signal , quickly locate roughly the search area, and then the search area in the new serial iterative search capture. The algorithm acquisition time is short, and it can capture the multipath energy, the number of iterations increases, the probability of detecting a signal corresponding to the number of stages will be improved, and the channel estimation can be more adapted to the existing UWB receiver receives .

\section{Adaptive Synchronization Acquisition Algorithm}

coarse synchronization acquisition algorithm. This paper describes the adaptive synchronization acquisition algorithms, the leading information communication is completed here by default. After the preamble the information is complete, we write the received signal is $T_{p}(t)^{[7]}$, correlation processing signals recorded as $T_{f}$,

$$
\begin{gathered}
T_{p}(t)=\sqrt{E_{p}} \sum_{i=0}^{+\infty} \sum_{j=0}^{N_{t}-1} q_{j} a_{j} p\left(t-i T_{f}-j T_{c}-T_{0}\right)+n(t) \\
=\sqrt{E_{p}} \sum_{i=0}^{+\infty} \sum_{j=0}^{N_{t}-1} q_{j} a_{j} p\left(t-\left(i N_{c}+j-T\right) T_{c}\right)+n(t) \\
T_{f}=N_{c} T_{c}=2^{N} T_{c}
\end{gathered}
$$

Capture algorithm is divided into coarse and fine synchronization capture two synchronous serial process. First performed coarse capture process , roughly quickly determine the search, constructed two local adaptive change template signals are related with the received signal . After making the comparison output, the search range is reduced to half of the original range, according to the algorithm continues to change the local signal quickly narrow your search. In the coarse synchronization acquisition basis, using a serial search method to capture the multipath components, determine the magnitude of the diameter and polarity .

$r_{p}^{2}(t)=E_{p} \sum_{i=0}^{+\infty} \sum_{j=0}^{N_{t}-1} a^{2}{ }_{j} p^{2}\left(t-\left(i N_{c}+j+T\right) T_{c}+2 \sqrt{E_{p}} \sum_{i=0}^{+\infty} \sum_{j=0}^{N_{t}-1} q_{j} a_{j} p\left(t-\left(i N_{c}+j+T\right) n(t)+n^{2}(t)\right.\right.$ 
(3)

Squaring the received signal, to reduce the effects of multipath channel due to the polarity of a certain degree. However, in order to more fully capture the energy of the diameter , the current algorithms are not apparent , thus combining the characteristics of UWB pulse signals, the number of the pulse signals can be considered as a standard combined adaptive reference signal.

$$
\begin{aligned}
& r_{i 1}(t)=\sum_{i=0}^{N-1} \sum_{j=0}^{n-i-1} p\left(t-k T_{f}-\sum_{n=0}^{i} \frac{\left(R_{(n-1) 0}<R_{(n-1))}\right) T_{f}}{2^{n-1}}-\tau_{1}\right), 1<i<M \\
& r_{i 2}(t)=r_{i 1}\left(t-T_{f} / 2^{i}\right)=\sum_{i=0}^{K-12^{k-i-1}-1} \sum_{j=0} p\left(t-k T_{f}-\sum_{n=0}^{i} \frac{\left(R_{(n-1) 10}<R_{(n-1) 1)}\right) T_{f}}{2^{n-1}}-T_{f} / 2^{i}-\tau_{i}\right), 1<i<M
\end{aligned}
$$

Wherein $\mathrm{N}$ is the signal length of the integral number of frames $; 2^{M-i}$ is the number of pulses during the iteration ; $\sum_{n=0}^{i} \frac{\left(R_{(n-1) 0}<R_{(n-1) 1}\right) T_{f}}{2^{n-1}}$ is the result of the previous iteration of the current feedback signal ; $\tau_{1}$ is the time constant.

By equation (4) and ( 5) we can deduce

$$
\begin{gathered}
\left(\sum_{n=0}^{i} \frac{\left(R_{(n-1) 0}<R_{(n-1) 1}\right) T_{f}}{2^{n-1}}-\tau_{1}\right)_{\lambda}=\left(\sum_{n=0}^{i} \frac{\left(R_{(n-1) 0}<R_{(n-1) 1}\right) T_{f}}{2^{n-1}}-\tau_{1}\right)_{\lambda-1}+\frac{\left(R_{(n-1) 0}<R_{(n-1) 1}\right) T_{f}}{2^{n-1}} \\
\left(R_{(n-1) 0}<R_{(n-1) 1}\right)= \begin{cases}1 & R_{(n-1) 0}<R_{(n-1) 1} \\
0 & R_{(n-1) 0} \geq R_{(n-1) 1}\end{cases} \\
R_{(n-1) 0}=\frac{1}{N} \int_{0}^{N T_{f}} r^{2}{ }_{p}(t) r^{2}{ }_{i 1}(t) d t \\
R_{(n-1) 1}=\frac{1}{N} \int_{0}^{N T_{f}} r^{2}{ }_{p}(t) r^{2}{ }_{i 2}(t) d t
\end{gathered}
$$

prep, $R_{(n-1) 0}, R_{(n-1) 1}$ is $r^{2}{ }_{i 1}, \quad r^{2}{ }_{i 2}$ and $r_{p}^{2}(t)$ after the output of the correlation, expressed in two different regions of the range of the received signal energy to the size , $\sum_{n=0}^{i} \frac{\left(R_{(n-1) 0}<R_{(n-1) 1}\right) T_{f}}{2^{n-1}}$ is the i-th iteration degree relative delay local template signal pulse position .

Coarse synchronization acquisition algorithm implementation :

(1) Search for $T_{f}$, and the initialization of each volume , adv. $R_{(n-1) 0}=R_{(n-1) 1}=0$, iterations $\mathrm{i}=0, \quad \sum_{n=0}^{i} \frac{\left(R_{(n-1) 0}<R_{(n-1) 1}\right) T_{f}}{2^{n-1}}=0$;

(2) Iterations $\mathrm{i}=\mathrm{i}+1$, by the formula (4) and (5), Derive the formula (8) and ( 9 ), the search area is divided into A, B two sections, then through judgment 
the size of the value $R_{(n-1) 0} 、 R_{(n-1) 1}$, determine the interval at which the search domain , the search time can be reduced by half;

(3) When the total number of iterations i is less than coarse synchronization iterations , return to step (2);

(4) When the total number of iterations $i$ is not less than the number of Coarse synchronization iterations $i_{c}$, Get the current search interval $\mathrm{X}$, and determines the success of coarse synchronization capture.

Fine synchronization acquisition algorithm. After the coarse synchronization capture is complete to get the search interval $\mathrm{X}$, on the need for fine synchronization , and this is the final result of the capture . Due to the presence of multi- path channel, coarse synchronization acquisition will be searched multiple synchronization signals within the range, Therefore, we need to add a timing parameter, To ensure that the signal energy obtained from the corresponding path is higher than the threshold $u$.The search interval is assumed that the received signal is:

$$
R_{g}=\{p: D(p) \geq u\}
$$

$D(p)$ is the size of the detection signal energy estimation value of the timing parameters when P. The impact of channel polarity can be taken local template signal with the received signal after squared correlation output, and then determine the magnitude relationship between the threshold value ,the search is determined to be greater than the target signal ,otherwise not, the search continues . After the search to get a search collection: $\left\{p_{i}: D\left(p_{i}\right)\right\}, p_{i}$ is multipath delay, $D\left(p_{i}\right)$ is the relative size of the i-path energy. This captures the energy of the strongest multipath channel signal information, while also channel estimation.

After the establishment of equations to establish the capture algorithm, due to the measured data tend to have some errors, so usually perturbation equations are obtained, which will make the fine synchronization capture more time-consuming , how to solve it?

Suppose $A x=b$

(1) Order $b$ 's disturbance is $\delta b$, Solve $x$ 's disturbance is $\delta x$.That equation (11) can be replaced with: $A(x+\delta x)=b+\delta b$

Then according to equation ( 11$)$ can be (12 ) simplifies to $: A \delta x=\delta b,\|\delta x\|=\left\|A^{-1} \delta b\right\| \leq\|A\|\|\delta b\|$

Wherein, $\mathrm{Z}$ is the length of the norm $\|A\|_{z}$,

$$
\|x\|_{z}=\left(\sum_{i=1}^{n} x_{i}^{2}\right)^{y_{2}} \text { and }\|x\| \geq \frac{\|A x\|}{\|A\|}=\frac{\|b\|}{\|A\|} \text {.so } \frac{\|\delta x\|}{\|x\|} \leq \frac{\|A x\| \delta b \|}{\frac{\|b\|}{\|A\|}}=\|A\|\left\|A^{-1}\right\| \frac{\|\delta b\|}{\|b\|}
$$


(2) Order $A$ 's disturbance is $\delta A$, solve $x$ 's disturbance is $\delta x$.That equation (11) can be transformed into : $(A+\delta A)(x+\delta x)=b$

According to equation ( 11$)$ ( 15$)$ can be simplified as follows: $A \delta x+\delta A(x+\delta x)=0$

So get $\|\delta x\|=\left\|A^{-1} \delta A(x+\delta x)\right\| \leq\left\|A^{-1}\right\| \delta A \|(\|x\|+\|\delta x\|)$

When $\delta A$ infinitely small, $\left\|A^{-1}\right\| \delta A \|<1$, get $\frac{\|\delta x\|}{\|x\|} \leq \frac{\left\|A^{-1}\right\| \mid\|A\|}{1-\left\|A^{-1}\right\| \delta A \|}$

Set cond $(A) \equiv\left\|A^{-1}\right\| A \|$ is a condition for the number of $\mathrm{A}$, The formula (13) error estimates are: $\frac{\|\delta x\|}{\|x\|} \leq \operatorname{cond}(A) \frac{\|\delta b\|}{\|b\|}$; Error of formula ( 18 ) is estimated as: $\frac{\|\delta x\|}{\|x\|} \leq \frac{\operatorname{cond}(A) \frac{\|\delta A\|}{\|A\|}}{1-\operatorname{cond}(A) \frac{\|\delta A\|}{\|A\|}}$. So cond $(A)$ more bigger, The greater the impact on the solution of the disturbance, This requires cond $(A)$ as small as possible. After obtaining approximation $x$,A generation back,Seek error value ( margin $\mathrm{r}$ ).If $\mathrm{r}$ is smaller, the higher the precision capture ${ }^{[8]}$.

\section{Detection Probability Analysis}

Coarse synchronization capture : the formula (5 ) into equation (8 ) can be obtained

$$
\begin{aligned}
& R_{(n-1)}(t)=\frac{1}{N} \int_{0}^{N T_{f}} r^{2}{ }_{p}(t) r^{2}{ }_{i 1}(t) d t=\frac{E_{p}}{N} \int_{0}^{N T_{f}} \sum_{i=0}^{+\infty} \sum_{j=0}^{M-1} b_{j}^{2} p^{2}\left(t-\left(i N_{n}+j+\tau\right) T_{f}\right) r^{2}{ }_{i 1}(t) d t \\
& +\frac{2 \sqrt{E_{p}}}{N} \int_{0}^{N T_{f}} \sum_{i=0}^{+\infty} \sum_{j=0}^{M-1} q_{j} b_{j} p\left(t-\left(i N_{n}+j+\tau\right) T_{f}\right) n(t) r^{2}{ }_{i 1}(t) d t+\frac{1}{N} \int_{0}^{N T_{f}} n^{2}(t) r^{2}{ }_{i 1}(t) d t
\end{aligned}
$$

Known previous iteration to the current signal feedback $\sum_{n=0}^{i} \frac{\left(R_{(n-1) 0}<R_{(n-1))}\right) T_{f}}{2^{n-1}}$ is an integer multiple of the $T_{f}$,prep $\sum_{n=0}^{i} \frac{\left(R_{(n-1) 0}<R_{(n-1) 1}\right) T_{f}}{2^{n-1}}=A_{i} T_{f}$

The formula (5) , (20) into 


$$
\begin{aligned}
& R\left(\tau: l, D_{p}\right)=\frac{E_{p}}{N} \int_{0}^{N T_{f}} \sum_{i=0}^{+\infty} \sum_{j=0}^{M-1} b_{j}^{2} p^{2}\left(t-\left(i N_{n}+j+\tau\right) T_{f}\right) \sum_{i=0}^{N-12^{N-1-1}-1} \sum_{j=0}^{2} p^{2}\left(t-j T_{f}-j^{\prime} T_{f}^{\prime}-D_{p} T_{f}\right) d t \\
& =\frac{E_{p}}{N} \int_{0}^{N T_{f}} \sum_{i=0}^{+\infty} \sum_{j=0}^{M-1} \sum_{i=0}^{N-12^{M-1-1}} \sum_{j=0} b_{j}^{2} p^{2}\left(t-\left(i N_{n}+j+\tau\right) T_{f}\right) p^{2}\left(t-\left(i N_{n}+j^{\prime}+D_{p}\right) T_{f}\right) d t
\end{aligned}
$$

Where $l$ is the channel gain, and can be introduced: $i N_{n}+j+\tau=l N_{n}+j^{\prime}+D_{p}$

Therefore, the formula ( $21 \quad)$ can be simplified to : $R\left(\tau: l, D_{p}\right)=E_{p} Q_{p}(0) \sum_{j=0}^{M-1} \tau_{j}\left(\tau: D_{p}\right) b_{j}^{2}$

prep $0 \leq j \leq M-1 ; 0 \leq \tau \leq N_{n}-1 ; 0 \leq D_{p} \leq N_{n} ; 2 M \leq N_{n} . Q_{p}(0)$ is $p^{2}(t)$ related handler, so $Q_{p}(0)=\int_{0}^{\infty} p^{2}(t) p^{2}(t+u) d t$. Equation can be obtained by the above formula ( 19 ) of the variance :

$$
\begin{gathered}
\sigma^{2}\left(\tau: l, D_{p}\right)=E\left[\left(\frac{2 \sqrt{E_{p}}}{N} \int_{0}^{N T_{f}+\infty} \sum_{i=0} \sum_{j=0}^{M-1} q_{j} b_{j} p\left(t-\left(i N_{n}+j+\tau\right) T_{f}\right) m(t) r^{2}(t) d t\right)^{2}\right] \\
=\frac{4 E_{p}}{N^{2}} E\left[n^{2}(t)\right]\left(\int_{0}^{N T_{f}+\infty} \sum_{i=0}^{M-1} q_{j=0} b_{j} p\left(t-\left(i N_{n}+j+\tau\right) T_{f}\right) r_{i}^{2}(t) d t\right) \\
=\frac{2 E_{p} Q_{p}(0)}{N} \sum_{j=0}^{M-1} \tau_{j}\left(\tau: D_{p}\right) b_{j}^{2}
\end{gathered}
$$

Which $m(t)$ is a Gaussian random variable. Then the output function can be related to $: Z_{p}=R\left(\tau: l, D_{p}\right)+m_{p}$

So you can get the promotion: $Z_{p 1}=R\left(\tau: l, D_{p}\right)+m_{p 1}$

$$
Z_{i 2}=R\left(\tau: l, D_{p}+2^{M-p}\right)+m_{p 2}
$$

In the coarse synchronization acquisition process, assuming the number of iterations is $\mathrm{n}$ times, the probability of the signal falls within the range of the search after the $\mathrm{m}$ is:

$$
P_{m}=\prod_{i, a_{i}=0}\left[1-O\left(-\frac{u_{i}}{\sigma_{i}}\right)\right] \cdot \prod_{j, a_{j}=0}\left(-\frac{u_{j}}{\sigma_{j}}\right)
$$

In the fine synchronization acquisition process, since the full search method is used, and therefore require multiple correlation detection. When given a threshold , you can get an average detection probability is: $P=E\left[\sum_{n=0}^{2^{n-1}} \sum_{i=0}^{2^{n-1}-1} P_{m} \bullet P\left(\gamma, d_{i, n}, j\right) \mid l, j\right]$

Can be obtained from equation ( 29) , to capture the pulse signal probability will become larger with the rapid increase in pulse signal to noise ratio, when the number of iterations increased, the capture probability will increase. But it is 
worth noting that captures the probability of this paper is based on multi -path energy loss and improve, so the number of iterations should be adequate to prevent the loss of multipath components of energy leads to increase in the error rate , but it can be done by source coding adding special symbol sequence of bits , so that the receiver can use these special bits for error correction to reduce the error rate.

\section{Capture time simulation results}

Coarse synchronization capture : The search area from $i=0$, every time the search , the search interval is reduced to half of the currently known interval until i $=N_{n}$, coarse synchronization acquisition search time: $T_{c}=N_{n} M T_{f}$, Wherein $M T_{f}$ is the integral time.

Fine synchronization capture : From the beginning of the search area $T_{c}$, the number of searches for : $N_{x}=R_{c} / 2^{N-N_{n}}$, Fine synchronization acquisition search time: $T_{x}=N_{x} O T_{f}$, Wherein $O T_{f}$ is the integral time.

The total acquisition time is: $T_{z}=T_{c}+T_{x}=\left(N_{n} M+2^{N-N_{n}} O\right) T_{f}$

By the formula ( 30 ) can be seen that the total acquisition time $T_{z}$ is related to $N_{n} 、 M T_{f} 、 O T_{f}$, Here 's $\mathrm{M}, \mathrm{O}$ is used to improve the SNR of noise suppression .

$$
\text { So : } N_{n}=N-\log _{2} J=\log _{2} N_{x}-\log _{2} J=\log _{2} \frac{N_{x}}{J}
$$

Normalized so that the disturbance in the foreseeable range control to minimize the error value ,order $\boldsymbol{M}=1 ; \boldsymbol{O}=1$, acquisition time are not considered here $T_{f}$, The average acquisition time with $\mathrm{J}$ the case where the path can be

calculated : $T_{p j}(J)=N_{n}+2^{N-N_{n}}=\log _{2} \frac{N_{x}}{J}+J$

At the same time, we can under the same conditions on the traditional linear acquisition algorithms, bit flipping capture algorithm, random capture algorithms, random replacement algorithm herein capture adaptive synchronization algorithm to capture the average acquisition time is calculated to make a comparison can be drawn from this paper since adaptive synchronization acquisition algorithm advantage in the average acquisition time .

Traditional linear acquisition algorithm mean acquisition time : $T_{p j}(J)=\frac{\left(N_{n}-J\right)^{2}+\left(3 N_{n}-J\right)}{2 N_{n}}$

$\begin{array}{crrr}\text { Bit-flipping } \quad \text { algorithm } & \text { captures mean } & \text { acquisition } \\ \text { time }: T_{p j}(J)=\frac{1}{2}\left(\frac{N_{n}}{J}+1\right) & & \text { (34) }\end{array}$ 


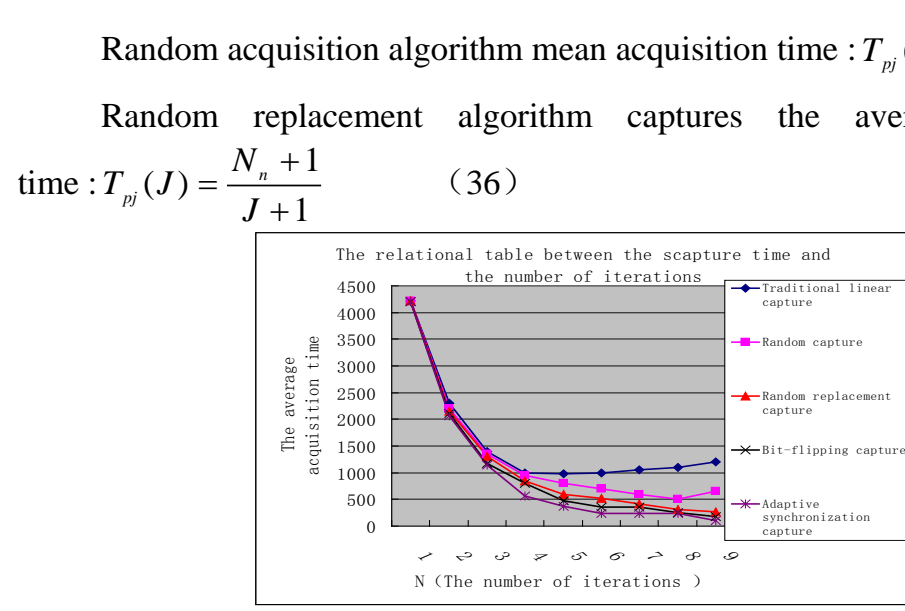

Figure1. The relationship between capture time

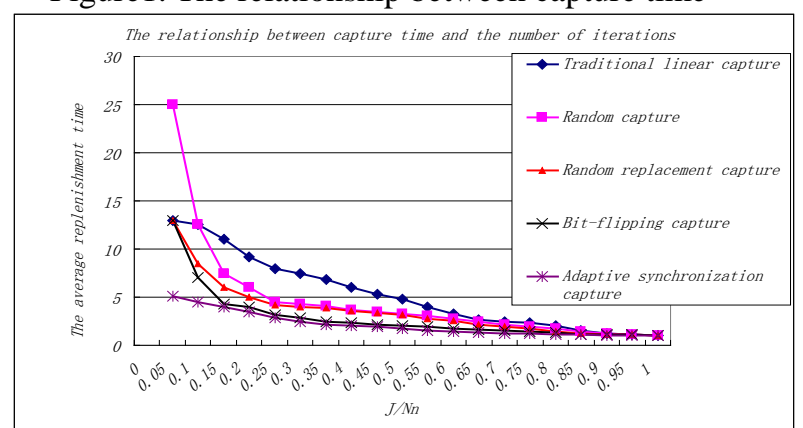

Figure2. The comparison table of average replenishment time and the number of iterations

Figure 1 is a five kinds capture algorithms capture time and the number of iterations of relational tables, we can see that the adaptive synchronization acquisition algorithm compared to other four kinds of algorithms increase in the number of iterations , the average time it takes to reduce the amount of capture is the largest ; Figure 2 is above average capture several acquisition algorithm and the proposed adaptive time synchronization algorithm to capture the average acquisition time simulation comparison chart. From Figure 2, it can clearly be seen that the proposed adaptive synchronization acquisition algorithms on the average acquisition time has a great advantage . Comprehensive Figure 1, Figure 2 , the adaptive synchronization acquisition algorithm in multipath environments synchronized capture the complexity of the average acquisition time at least , the effect is optimal.

\section{Summary}

Multi- path channel environment, UWB synchronization has been a very complex issue. This paper presents an adaptive synchronization acquisition 
algorithms, fully overcome the disadvantages of multi- path channel , able to quickly capture the sync signal, and obtain the relevant synchronization information. At present, the research synchronous capture technology are many, but the relevant proposed algorithm compared to the capture of several known

algorithms still have a great advantage in the average acquisition time and channel estimation on the advantages more apparent .

\section{References}

[1] Jeongwoo Han, Cam Nguyen, A new ultra-wideband.ultra-short monocycle pulse generator with reduced ringing, IEEE Microwave and Wireless Components Letters, Volume: 12, Issue: 6 , 2002, pp.10- 20.

[2] W.M.Lovelaee, J.K.Townsend, The effects of timing jitter on the Performance of impulse radio[J],IEEE Joumal on Selected Areas in Communications, Volume:20, 2002, pp.9.

[3] Z.Tian,G.B.Giannakis.BER, Sensitivity to mistiming correlation based UWB receivers, Proceedings IEEE Globeeom Conference, SanFrancisco, 2003, pp.9- 17.

[4] Z.Tian and G.B.Giannakis.Ber, Sensitivity to mistiming in ultra-wide band impulse radios-Part 11:fading channels, IEEE Transactions on Signal Processing, 2005, pp.1897--1907.

[5] F. Ramirez-Mireles, On performance of ultra-wideband signals in Gaussian noise and dense multipath, IEEE Transactions on Vehicle Technologies, 2001, pp.244-250.

[6] Peng Wang, UWB wireless communication system synchronization techniques, master's thesis, Shandong University ,2008,pp.32-33.

[7] Yongwei Qiao, Synchronization and demodulation algorithm for ultra-wideband (UWB) wireless communication system, PhD Thesis, Beijing University of Posts and Telecommunications, 2009,pp.40-41.

[8] Qinyang Li, Nengchao Wang, Dayi Yi, Numerical Analysis, Publisher: Tsinghua University Press, 2008,pp.191-197. 